\title{
Dynamic Teaching of Kinematics of Particles and Python
}

\author{
Ting-Sheng Weng, Meng-Hui Hsu, and Der-Ching Yang
}

\begin{abstract}
The objective of this study is to apply the open source code Python and industrial software MATLAB on the kinematic analysis of a particle with golf or Projectile motion. By achieve this purpose. we integrate the kinematic analysis of the particle with programming capability. It is found that the computer dynamic simulation enables the students to know the representation of kinematics of particles, and realize the graphic representation of basic physical concepts of golf or Projectile motion. The dynamic teaching allows the students to better understand different effects of different curved surfaces, and it is advantageous for the students to know basic academic theories of physics.
\end{abstract}

Index Terms-Kinematic analysis, open source code, dynamic simulation.

\section{ReSEARCh Motives AND PURPOSES}

The kinematics of particles is one of basic studies of physics, and it is extensively used in the phenomena of daily life, such as free falling body and Projectile motion. However, the traditional teaching lays emphasis on calculation and deduction of equations, while neglects whether the students know the physical states. In addition, in the kinematics of particles, the rectilinear motion is easier for students to understand than curvilinear motion, because the angle problem should be considered in curvilinear motion. In general traditional teaching, the angular difference cannot be presented precisely, thus, how to let the students comprehend the principles and build the links between principles and practical phenomena during learning is the motive of this study. This study uses freeware python to develop auxiliary teaching animation to help the students creating the links between the concept of kinematics of particles and actual phenomena. The dynamic effect of kinematics of particles is present for students to learn the meanings of physical equations. The dynamic graphics-based teaching mode can help to enhance the students' comprehension and relevant knowledge effectively, and to assist the students in strengthening the capability for computer information. As a result, the students can utilize the knowledge they learnt effectively in future works.

The images of this auxiliary teaching animation must present theorematic equations and 3D graphics, and the students can find that different equations result in different graphics and interparticle changes from the modification of program code.

Therefore, this study develops the auxiliary teaching animation containing the following functions:

1) Equation display area

Manuscript received April 19, 2013; revised June 25, 2013.

Ting Sheng Weng is with the National Chiayi University, Taiwan (e-mail: politeweng@mail.ncyu.edu.tw).
2) 3D picture display area

3) Function of particle position change

\section{LITERATURE REVIEW}

\section{A. Kinematics of a Particle}

Chen and Hsu [1] indicated that one object in any uniform ideal fluid in even depth, the wave resulted from free surface gravity with irrotational motion would fluctuate. In Eulerian and Lagrangian computing modes, if all the corresponding basic governing equations are met, the solution must be obtained through step-by-step operating mode, thus obstructing the students' learning, the assistance of dynamically visual effect will be helpful to the absorbability of students for learning.

Wei and Hsieh [2] suggested that the angular velocity could be divided into four angular velocity periods and non-angular velocity periods. The angular velocity period contains acceleration period, constant velocity period, deceleration period and peak accelerations of various periods. Each accelerating period represents a physical phenomenon. The velocity of physical effect is violent in the acceleration period, different acceleration characteristics transformed by the variation in weight can be observed when the object is in the acceleration period. This characteristic can be used to analyze the acceleration effect and conversion process of different objects. This study discusses different velocities generated when the plane torsion of curvature makes the smooth bearer roll onto the plane.

Yang, Ho and Kao [3] found that different materials or volumes have different stress effects. When the volume is large, the stress is high, suggesting that the degree of disturbance is greater than small objects. In the motion on general curved surface, the effect of small volume is direct. In this study, these physical properties are used to present the dynamic stress situation by Python drawing software, which is very helpful to teaching or designing products, especially the cases of different angles are inconsistent. The vision-assisted effect representation is helpful to comprehension and analysis of students.

\section{B. Dynamic Teaching}

Cornwell [4] argued that despite of teachers' efforts in teaching the meanings of physical properties, many students still rely on numerical calculation and equations to find solutions, without truly understanding the application of physical problems. Therefore, the dynamic teaching is expected could help the students using their imagination and developing dynamic intuition.

Lin [5] used the teaching material of dynamic pictures to annotate abstract mathematical concepts, such as synthesis of continuous function and graphic solution of derivative, are 
helpful to the comprehension and calculation of abstracts. Moreover, it could be used as assistant teaching data for students in teaching, so as to reduce the obstacles in the students' learning.

Nishizawa and Yoshioka [6] indicated that the students emphasize on memorizing equations and solution calculation in mathematical learning, but lack comprehension of graphics. The dynamic graphic software can be used to interpret 2D and 3D graphics. As the interpreter is students instead of teachers, better learning effect can be achieved.

\section{RESEARCH METHOD}

The physical characteristics are combined with simulation effect to present the dynamic procedure of Golf and Projectile motions. The dynamic simulation of 3D Golf motion is executed by Python software, and the dynamic simulation of Projectile motion is executed by MATLAB software. Then, the two motions are used in teaching to observe the learning motivation and reaction and thinking abilities of students.

\section{A. Example 1 (Golf Motion)}

G. Galileo found that all the free falling bodies, without reference to weight and size, they moved with uniform acceleration somewhere about the earth surface, and all of falling bodies in the same place on the earth had the same acceleration. The direction of free-fall acceleration is downward along vertical direction, its magnitude is usually represented by $\mathrm{g}$, and the $\mathrm{g}$ value is generally called gravitational acceleration. Therefore, in the program, the vector of velocity is set as vector $(1,-1,0)$, i.e. $x=1$, $y=-1$ falling off, the trajectory of golf rebound is determined by kinetic equation of $\sin (x)+i \cos (x)$. The freeware python is used to design program to show the states of golf motion. The program codes are described below:

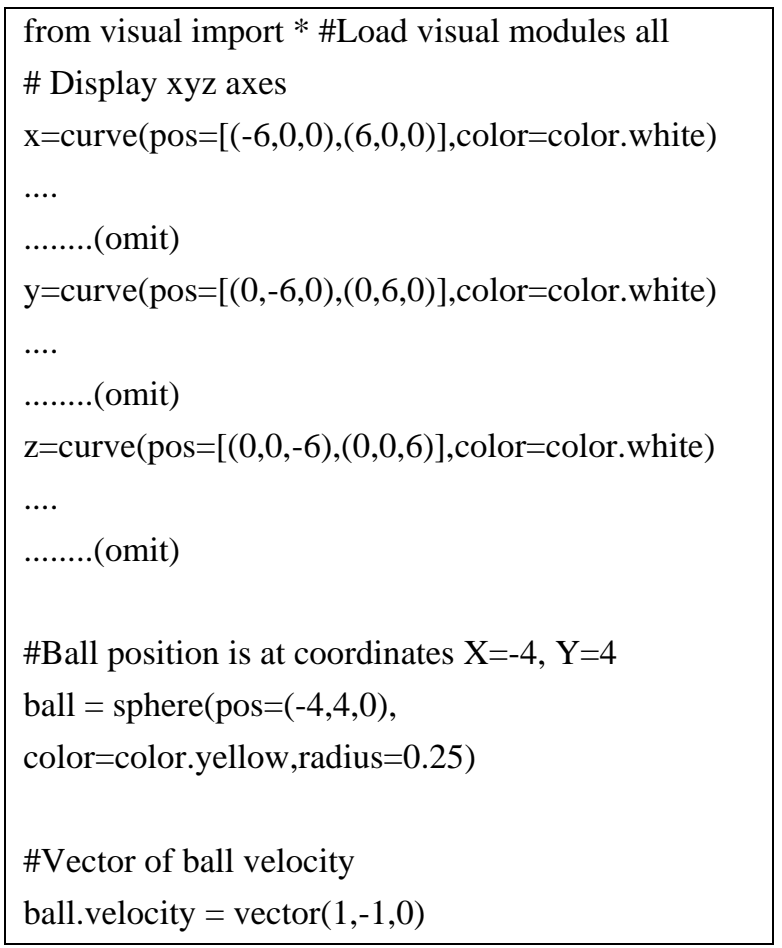

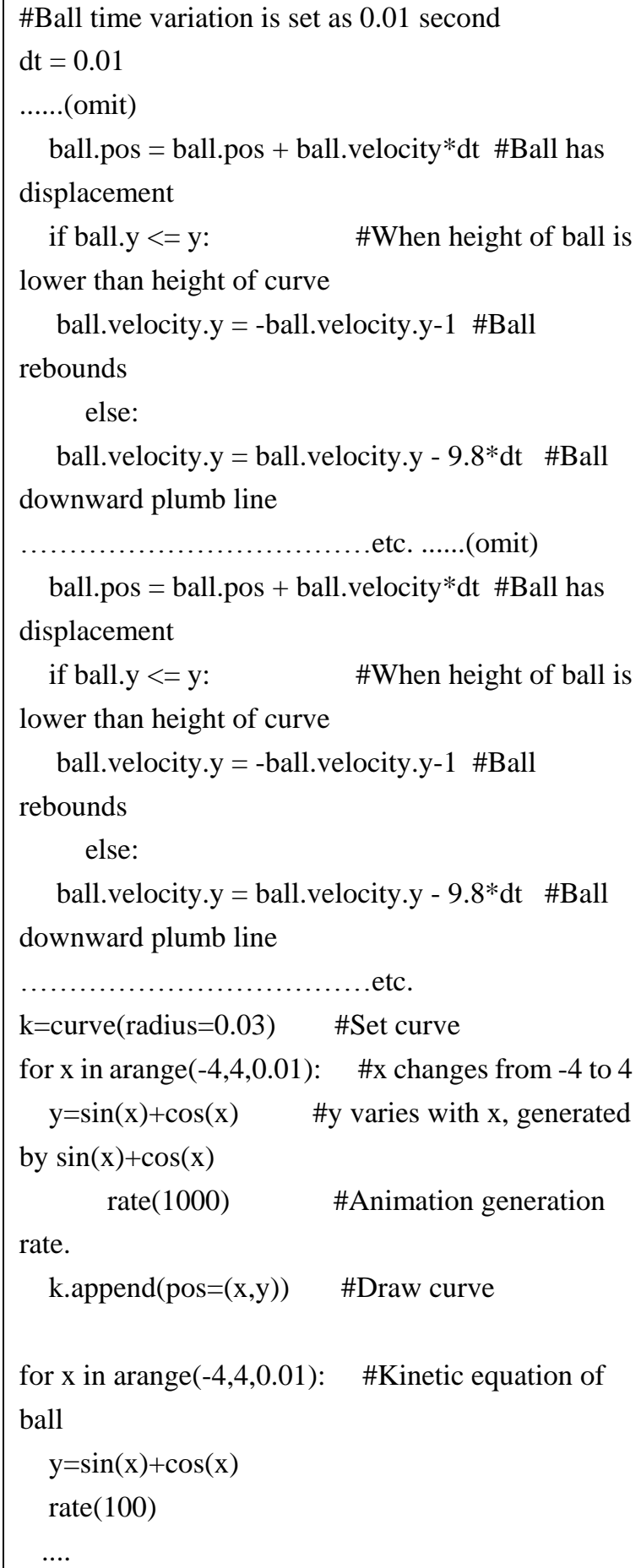

ball.pos = ball.pos + ball.velocity*dt \#Ball has displacement

if ball.y <=y: \#When height of ball is

lower than height of curve

ball.velocity.y = -ball.velocity.y-1 \#Ball rebounds

else:

ball.velocity.y = ball.velocity.y - 9.8*dt \#Ball downward plumb line

...........................etc.

$\mathrm{k}=$ curve(radius=0.03) \#Set curve

for $\mathrm{x}$ in arange $(-4,4,0.01)$ : \#x changes from -4 to 4

$\mathrm{y}=\sin (\mathrm{x})+\cos (\mathrm{x}) \quad \# \mathrm{y}$ varies with $\mathrm{x}$, generated

by $\sin (\mathrm{x})+\cos (\mathrm{x})$ rate(1000) \#Animation generation rate.

k.append $(\operatorname{pos}=(\mathrm{x}, \mathrm{y})) \quad$ \#Draw curve

for $\mathrm{x}$ in arange(-4,4,0.01): \#Kinetic equation of ball

$\mathrm{y}=\sin (\mathrm{x})+\cos (\mathrm{x})$

rate(100)

....

Fig. 1 to Fig. 3 show the dynamic simulation of 3D Golf motion executed by Python software.

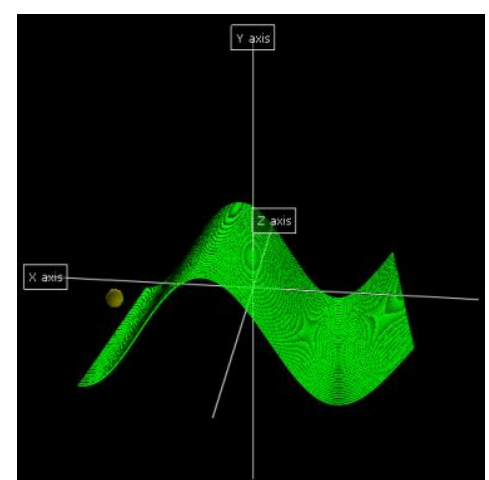

Fig. 1. Ball motion positions observed at different angles. 


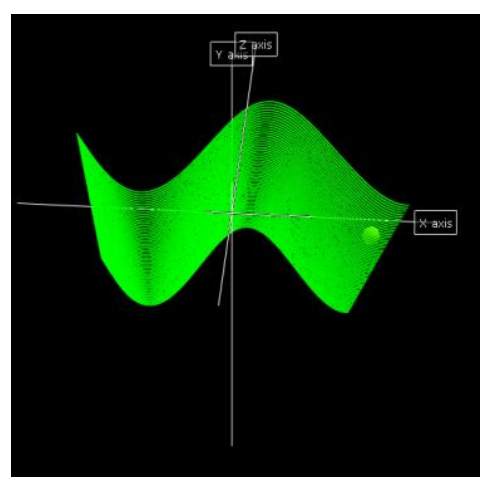

Fig. 2. Ball motion landing points observed at different angles.

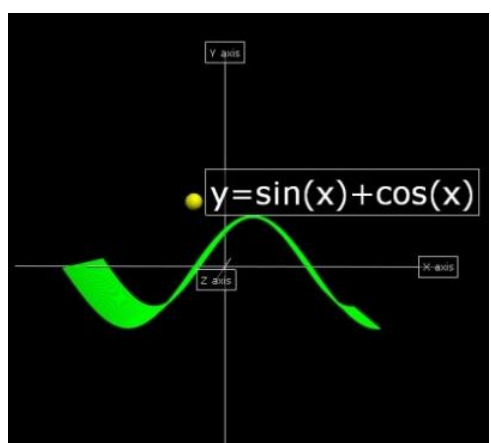

Fig. 3. Window picture shows mathematical formula for students' comprehension.

\section{B. Example 2 (Projectile Motion)}

Projectile motion is an example of parabolic curved motion with constant acceleration and is shown in Figure 4.

The starting point $\mathrm{O}$ of projectile motion is the origin of rectangular coordinates, the horizontal and vertical coordinate axes are $\mathrm{x}$-axis and $\mathrm{y}$-axis. $v_{0}$ is the initial velocity of projectile motion, $\theta_{0}$ is the included angle between initial velocity and horizontal direction. The kinematic analysis of a particle projectile motion is described as below:

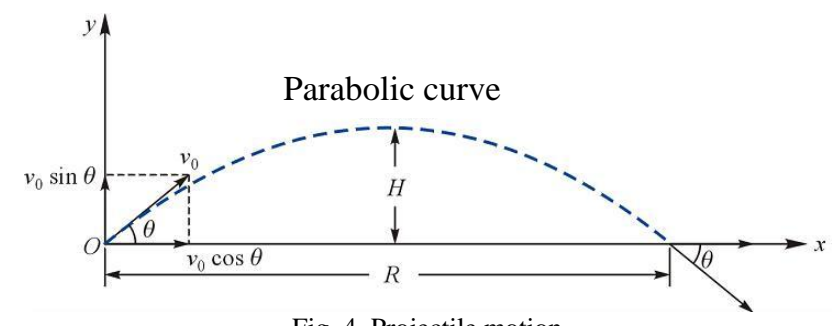

Fig. 4. Projectile motion.

\section{Vertical Motion}

As it is downward motion with constant gravitational acceleration $g$, so the vertical velocity equation is:

$$
v_{y}=v_{0} \sin \left(\theta_{0}\right)-g t
$$

Vertical displacement equation is:

$$
y=v_{0} \sin \left(\theta_{0}\right) t-\frac{1}{2} g t^{2}
$$

\section{Horizontal Motion Equation}

The horizontal velocity component is constant, i.e.:

$$
v_{x}=v_{0} \cos \left(\theta_{0}\right)
$$

Horizontal displacement equation is:

$$
\begin{gathered}
x=v_{x} t=v_{0} \cos \left(\theta_{0}\right) t \\
t=\frac{x}{v_{0} \cos \left(\theta_{0}\right)}
\end{gathered}
$$

According to Eq. (2) and Eq. (5), the trajectory of the particle is a parabolic curve:

$$
y=\tan \left(\theta_{0}\right) x-\frac{1}{2} \frac{g}{\left[v_{0} \cos \left(\theta_{0}\right)\right]^{2}} x^{2}
$$

If the vertical position of particle is zero, it means that the particle falls to the ground, so according to Eq. (2):

$$
0=v_{0} \sin \left(\theta_{0}\right) t-\frac{1}{2} g t^{2}=t\left[v_{0} \sin \left(\theta_{0}\right) t-\frac{1}{2} g t\right]
$$

The initial time $t_{0}=0$ or landing time $t_{1}=\frac{2 v_{0} \sin \left(\theta_{0}\right)}{g}$ can be known. Therefore, the maximum horizontal displacement $R$ :

$$
R=v_{0} \cos \left(\theta_{0}\right) t_{1}=\frac{v_{0}^{2} \sin \left(2 \theta_{0}\right)}{g}
$$

Maximum vertical displacement $H$ :

$$
H=\tan \left(\theta_{0}\right) \frac{R}{2}-\frac{1}{2} \frac{g}{\left[v_{0} \cos \left(\theta_{0}\right)\right]^{2}}\left[\frac{R}{2}\right]^{2}=\frac{\left[v_{0} \sin \left(\theta_{0}\right)\right]^{2}}{2 g}
$$

According to Eq. (5) and Eq. (7), we have $0 \leq t \leq \frac{2 v_{0} \sin \left(\theta_{0}\right)}{g}$.

Based on the mathematical Equations (1) to (7), we used the software MATLAB to design program for showing the states of projectile motion. The program codes are described below:

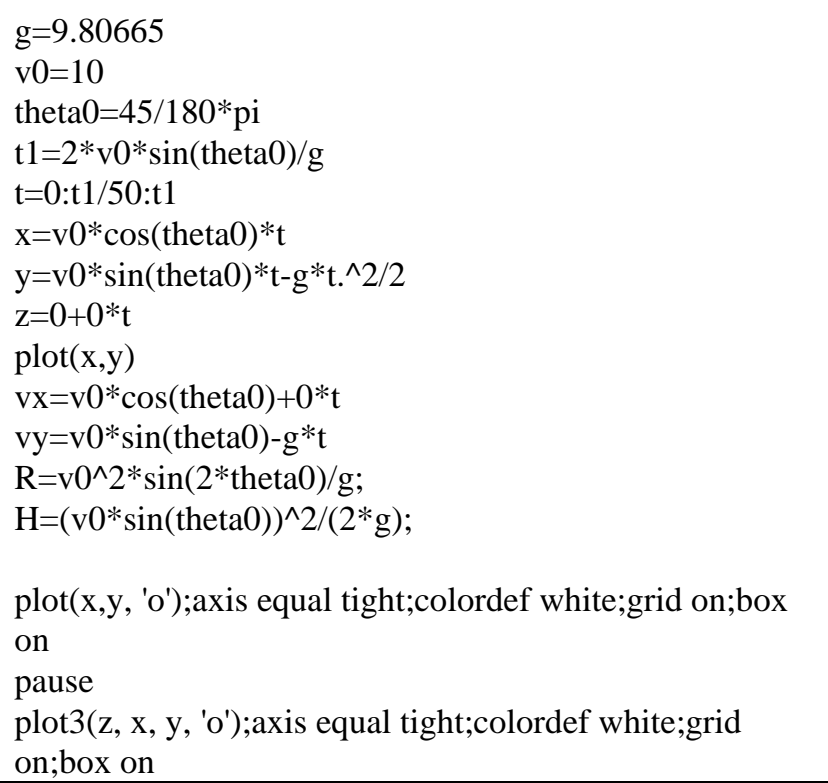


Fig. 5 and Fig. 6 show the dynamic simulation of 2D and 3D projectile motion executed by MATLAB software.

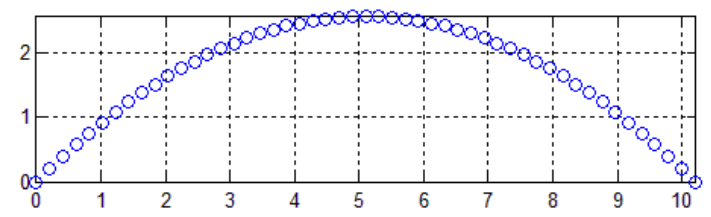

(a) 2D Parabolic curve

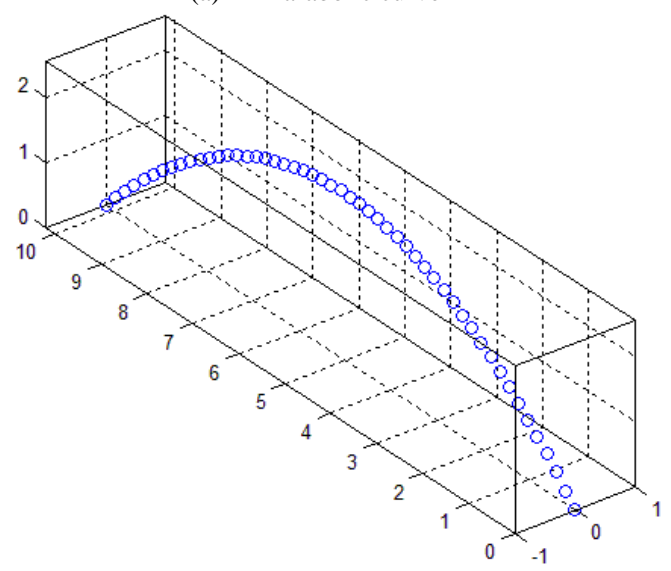

(b) 3D Parabolic curve

Fig. 5. Projectile motion $\left(\theta_{0}=45^{\circ}, v_{0}=10 \mathrm{~m} / \mathrm{s}, H=2.5493 \mathrm{~m}\right.$, $R=10.1972 m)$.

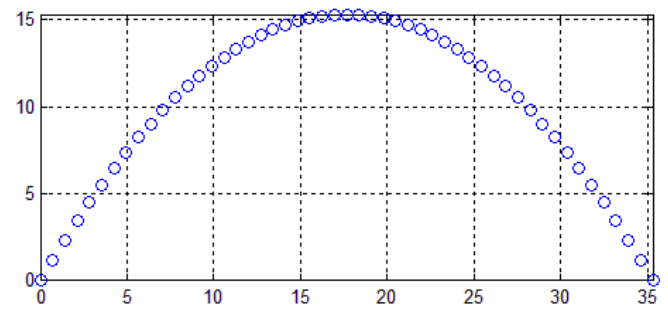

(a) 2D Parabolic curve

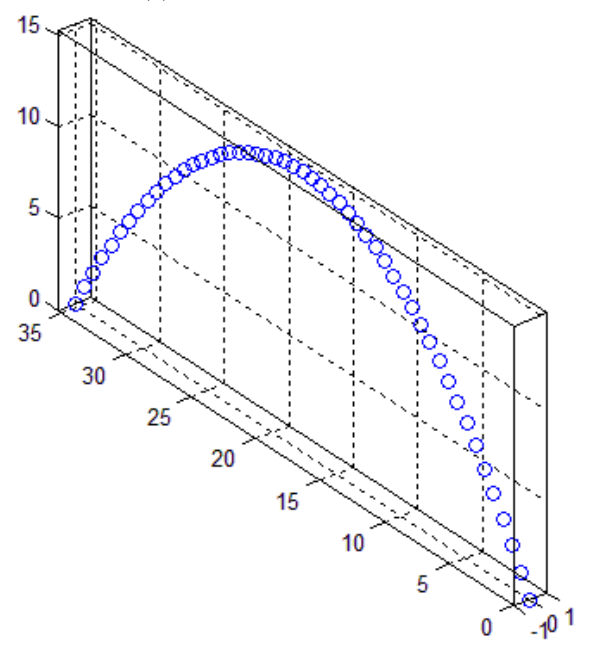

(b) 3D Parabolic curve

Fig. 6. Projectile motion $\left(\theta_{0}=60^{\circ}, v_{0}=20 \mathrm{~m} / \mathrm{s}, H=15.2957 \mathrm{~m}\right.$, $R=35.3240 m)$.

\section{CONCLUSION}

The particle motion state can be presented by using freeware python and industrial software MATLAB. When it is applied to teaching, the students can know the physical phenomenon of kinematics of a particle, and further understand the principle of physical phenomenon equation from the application of software program codes. They can use this ability in more different mathematical concepts, thus building confidence in learning mathematical studies. In terms of popularization, this technology can be used to simulate the projectile trajectory of golf and Projectile motion, and the kinematic trajectory of the moving particle can be simulated at different projecting angles.

\section{ACKNOWLEDGEMENT}

The authors appreciate the comments of the review committee. This project was supported by the National Science Council under Grant No.NSC101-2511-S-415 -006.

\section{REFERENCES}

[1] Y. Y. Chen and H. C. Hsu, "The Transformation between the Third-Order Eulerian and Lagrangian Solutions for Irrigational Standing Gravity Waves," Journal of Coastal and Ocean Engineering, vol. 8, No. 2, pp. 93-121, 2008.

[2] S. H. Wei and H. H. Hsieh, "Mechanical Analyses of Acceleration during Transition Period of Knee Isokinetic Exercises," Journal of the Physical Therapy Association of the R.O.C., vol. 23, no. 2, pp. 83-89, 1998.

[3] S. C. Yang, C. E. Ho, and C. R. Kao, "The Effect of Volume on the Soldering Reaction between SnAgCu Solders and Ni," Journal of Materials Science and Engineering, vol. 37, no. 4, pp. 230-234, 2005.

[4] P. J. Cornwell, "Teaching Dynamics Using Modern Tools," Computers in Education Journal, Oct.-Dec. 1996.

[5] Y. C. Lin, "Calculus Teaching and Dynamic Teaching Material Design," Journal of General Education, no.5, pp.171-191, 2003.

[6] H. Nishizawa and T. Yoshioka, "A Proposal to Teach 3D Vector Operations in a Role-Playing Game," in Proc. the 13th Asian Technology Conference in Mathematics, 2008.

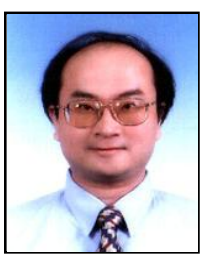

Ting Sheng Weng is an assistant professor of the department of Business Administration at National Chiayi University, Taiwan. He received his $\mathrm{Ph}$. D. in Division of management and information science, Graduate School of Advanced Technology from Kinki University, Japan in 2000. He had served as visiting Professor at Meiji University in Japan from 2006 through 2007. His major research interests include science technology and management, e-learning, Information Education, Technology Education, MIS, and image processing.

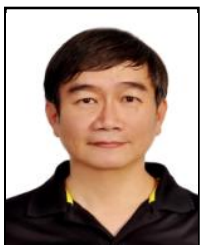

Meng-Hui Hsu took M. S. and $\mathrm{Ph}$. D. from the Department of Mechanical Engineering, National Cheng-Kung University, TAIWAN, 1990 and 1996 , respectively. Now Dr. Hsu is an associate professor of Mechanical Engineering Department at Kun-Shan University in TAIWAN. His research interests include Mechanism and Machine Design, Theory of Creative Design, and Servo Control Systems.

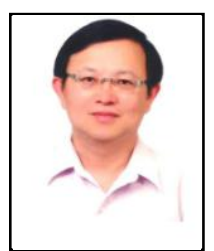

Der-Ching Yang is a professor of the Graduate Institute of .Mathematics and Science Education at National Chiayi University. Dr. Yang received his PhD in mathematics Education from University of Missouri-Columbia in 1995. His research focuses on number sense, mathematics teaching \& learning, technology \& mathematics teaching and learning, curriculum study. 